\title{
Effect of required response force on rats' performance on a VI+ schedule of reinforcement
}

\author{
PHIL REED \\ Swansea University, Swansea, Wales
}

\begin{abstract}
Three experiments were performed to examine the effect of response force on rats' performance on various schedules of reinforcement. Response force was manipulated by changing the weight of the lever in the operant chamber-a heavy lever for high response force and a light lever for low response force. Using a within-subjects design, Experiment 1 replicated previous findings that rats respond more quickly on variable ratio (VR) than on equivalent variable-interval-plus-linear-feedback (VI+) schedules. Experiment 2 replicated this finding but also showed that the use of a smaller response force abolished the response rate difference between the VR and VI+ schedules. Experiment 3 used a between-subjects design and showed a response rate difference between the VR and VI+ schedules with a high response force but no response rate difference with a low response force. This suggests that under conditions of low force, when the rats' responding can continue at prolonged high rates, these subjects show little difference in their response rates between VR and VI+ schedules. These data are similar to those found for human subjects.
\end{abstract}

McDowell and Wixted (1986) introduced a schedule that has the molar properties of a variable ratio (VR) schedule (i.e., a strong function relating response rate to reinforcement rate) but the molecular properties of a variable interval (VI) schedule (i.e., one that differentially reinforces long interresponse times [IRTs]). This schedule can be called the variable-interval-plus-linear-feedback (VI+) schedule. This contingency reinforces high rates of responding by making rates of reinforcement depend on rates of responding. The VI+ schedule achieves this by making the interval required for reinforcement vary inversely with response rate. However, the VI+ schedule also maintains the interval characteristics of the VI schedule that differentially reinforces long IRTs.

Figure 1 shows a schematic representation of the feedback function between response rate and reinforcement rate for three schedules (VR, VI, and VI+). The feedback function of the VI+ schedule is given by the following equation: $a=(i / n) * b$. Here, $a$ is the interval required before a response will produce reinforcement, $i$ is the time since the last reinforcement, $n$ the number of responses emitted during that period, and $b$ the VR ratio to which the VI+ schedule is equivalent. Some numeric examples may serve to illustrate how this schedule works. Assume that the VI+ schedule was to have the molar characteristics of a VR-30 schedule. Assume also that the time from

These data were first presented at the Fourth European Meeting for the Experimental Analysis of Behaviour, Amiens, France, 2000. Thanks are due the University of Leeds for providing laboratory space and Lisa A. Osborne for her support. Correspondence concerning this article should be addressed to P. Reed, Department of Psychology, University of Wales Swansea, Singleton Park, Swansea SA2 8PP, England (e-mail: p.reed@ swansea.ac.uk). the last reinforcer was $60 \mathrm{sec}$ and that 60 responses had been made during that time. In this case, the interval to reinforcement would equal $(60 / 60) * 30=30 \mathrm{sec}$. During this $30 \mathrm{sec}$, at 60 responses per minute, 30 responses would be emitted before the interval would time out. If 30 responses had been made during that 60 -sec period, the interval would be $(60 / 30) * 30=60 \mathrm{sec}$. During this $60 \mathrm{sec}$, if responses were being emitted at 30 responses per minute, 30 responses would be emitted prior to the reinforcer. Alternatively, if 120 responses had been made during the 60 -sec period, the interval would become $(60 / 120) * 30=$ $15 \mathrm{sec}$. During this $15 \mathrm{sec}$, at 120 responses per minute, 30 responses would be emitted prior to the reinforcer. Thus, the interval varies inversely with the rate of responding, and each reinforcer is delivered for about 30 responses.

McDowell and Wixted (1986) found that with human subjects, high response rates were produced by VR schedules and also by VI+ schedules that had the same feedback functions as the VR schedules. Reed (in press) replicated and extended the findings reported by McDowell and Wixted with human subjects. Reed (in press) noted that their human subjects responded at a similar rate on VR and VI+ schedules and more quickly on these schedules than on VI schedules yoked to the former contingencies in terms of rate of reinforcement. Importantly, the latter study indicated that subjects were more sensitive to the molar aspects of the VI+ schedule when they were given a smaller response force requirement than that used by McDowell and Wixted.

In contrast to these results reported with human subjects, three studies have addressed performance on VI+ schedules, using rat subjects (Cole, 1999; Reed, Hildebrandt, DeJongh, \& Soh, 2003; Reed, Soh, Hildebrandt, DeJongh, \& Shek, 2000), and none of these studies showed 

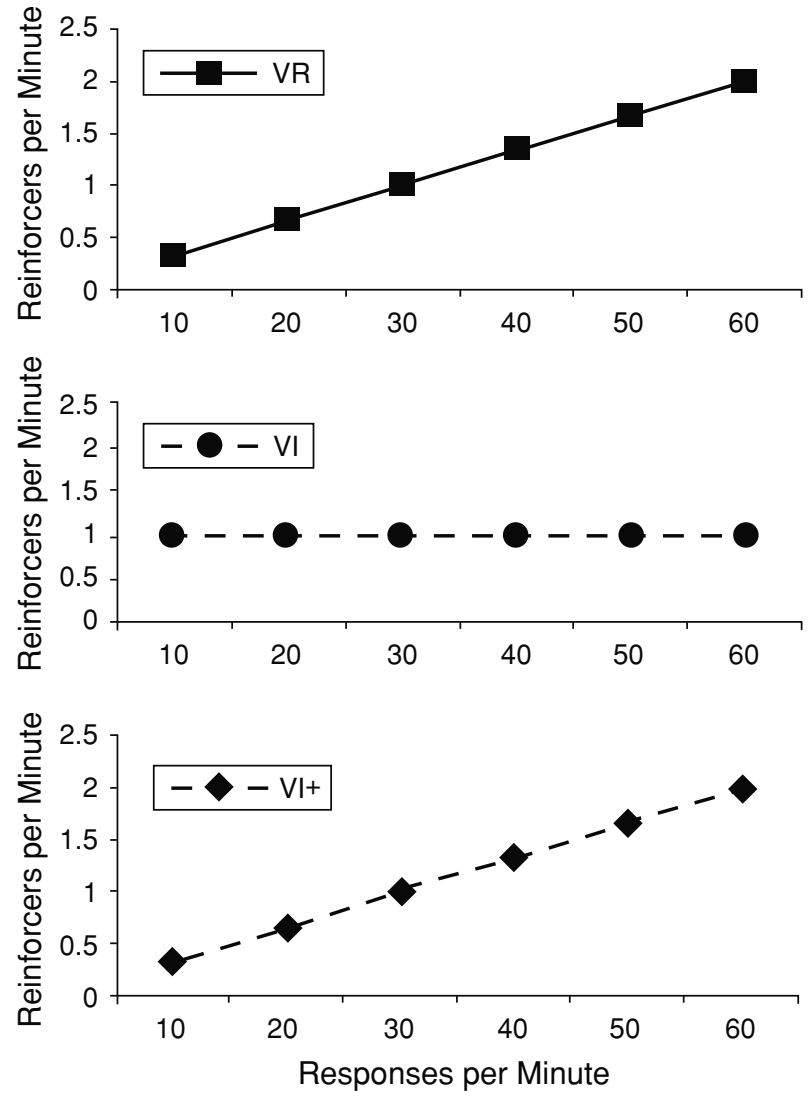

Figure 1. Schematic representation of the feedback functions relating rate of responding to rate of reinforcement for variable ratio (VR) 30, variable interval (VI) $60 \mathrm{sec,} \mathrm{and} \mathrm{variable} \mathrm{interval}$ with linear feedback (VI+) 30.

that the VI+ schedule supported rates of responding as high as those on the VR schedule. In fact, Cole actually found that the VI+ schedule was incapable of supporting behavior in some rats. All of these studies (Cole, 1999; Reed et al., 2003; Reed et al., 2000) showed that the VI and VI+ schedules produced similar reinforced IRTs that were nearly four times longer than those produced by the VR schedule. This reinforced IRT data, in itself, offers an explanation of the response rate data obtained in Cole's study. Reed et al. (2003; Reed et al., 2000) experimentally manipulated the effects of reinforced IRTs by yoking the reinforced IRTs on a VI+ schedule to those on a VR schedule. Only under these circumstances were response rates on the VI+ schedule similar to those on a VR schedule. Thus, none of these studies showed compelling evidence of any impact of the molar aspects of the VI+ contingency on rats' performance.

The present study employed one of the findings noted in Reed's (in press) study with humans in order to ascertain whether there were conditions under which rats would show sensitivity to the molar aspects of the VI+ contingency. In particular, it might be that the rats would show sensitivity to the molar properties of the VI+ schedule, if the force required to depress the lever were made very small. There were a number of reasons to suggest that this might be the case. The rates of responding generated by the human subjects, who did show sensitivity to the molar aspects of the VI+ schedule, were much higher than those noted for the rats, which did not show sensitivity (cf. Cole, 1999; McDowell \& Wixted, 1986; Reed, in press; Reed et al., 2003; Reed et al., 2000). Reducing the force required to make a response may allow rates of responding in rats to increase (see Kurtz, 1970; Sumpter, Temple, \& Foster, 1998). Such high rates may be important, since Cole (see also Reed et al., 2000) suggested that the functioning of the VI+ schedule makes any pause in responding potentially catastrophic for local reinforcement rates. A reduction in rate of responding on the $\mathrm{VI}+$ schedule will generate an increased interreinforcement interval, which, if it becomes too long, will lead to extinction (see the data in Cole, 1999; Reed et al., 2003). If rates are not maintained across the schedule, this can dramatically reduce response rates seen on the VI+ schedules.

The present series of experiments manipulated response force in order to make responding at high rates easier for the rats. Conditions that allow prolonged emission of high rates of response in rats might allow a better comparison with the situation noted for human subjects. If this were the case, rats with a low force requirement should show little difference in response rates between the VR and the VI+ schedules, as has been noted with human subjects (see McDowell \& Wixted, 1986; Reed, in press). In contrast, rats responding with a higher force requirement should show VR response rates that outstrip those seen on the VI+ schedule (see Cole, 1999; Reed et al., 2003; Reed et al., 2000). Such a result would demonstrate a functional equivalence between the performance seen on the VI+ schedule across species under the appropriate conditions.

\section{EXPERIMENT 1}

In the first experiment, a novel within-subjects technique was used to assess sensitivity to the molar aspects of the $\mathrm{VI}+$ schedule. Rats were trained on a multiple VR-30 VI+ schedule, with the feedback function of the VI+ schedule made to equate that of the VR schedule. Since this was a new procedure, the aim of the first experiment was to demonstrate that under the response force conditions previously employed with between-subjects designs (i.e., Reed et al., 2003; Reed et al., 2000), there would be a higher response rate on a VR schedule component than on a VI+ schedule component.

\section{Method}

Subjects. Eight male Sprague Dawley rats served in the present experiment. The subjects were 7 months old at the start of training, had a free-feeding body weight range of 390-505 g, and were maintained at $85 \%$ of this weight throughout the experiment. The subjects had previously served in an appetitive classical conditioning experiment, during which they had been exposed to lights, tones, and food, but they were naive with respect to leverpressing and to all the schedules that were used in the experiment. The subjects were housed in groups of 4 , with water constantly available in the home cage. 
Apparatus. Four identical operant conditioning chambers (Campden Instruments Ltd.) were used. Each chamber measured $23.5 \times 23.5 \times 20 \mathrm{~cm}$ (length $\times$ width $\times$ height). Each chamber was housed in a light- and sound-attenuating case, ventilated by a fan that provided background masking noise (65 db [A]). Each chamber had two retractable levers, positioned either side of a centrally located food hopper. The hopper was covered by a hinged clear Perspex flap. There were jeweled houselights above each of the levers. Reinforcement consisted of one 45-mg food pellet and was delivered to the food hopper.

Procedure. The subjects received 2 sessions of magazine training on a variable time (VT) 60 -sec schedule, with both levers retracted from the chamber. They then received two 20 -min sessions of leverpress training on a continuous reinforcement (CRF) schedule. In one session, the left lever was used, and in the other session, the right lever was used (the alternate lever was withdrawn from the chamber during these sessions). All the subjects were then transferred to a mult VR, VR schedule for 14 sessions. Each of these sessions lasted for $60 \mathrm{~min}$. The components of the multiple schedule were presented six times each during a session, in a random order. A component would be signaled by the insertion of one lever into the chamber and also by the illumination of the houselight above that lever. The inoperative lever remained withdrawn from the chamber, and the houselight above this lever remained extinguished. Each component lasted for $5 \mathrm{~min}$, with a 30 -sec intercomponent interval. During the intercomponent interval, both levers were withdrawn, and both houselights were extinguished.

Over the course of pretraining, the VR value was gradually increased as follows: Session 1, mult VR-5, VR-5; Session 2, mult VR-10, VR-10; Sessions 3-5, mult VR-15, VR-15; Sessions 6-9, mult VR-20, VR-20; Sessions 10-14, mult VR-25, VR-25.

Following pretraining, all the subjects were exposed to a mult VR-30, VI+ schedule. The feedback function relating the rate of response to the rate of reinforcement on the VI+ schedule was the same as that on a VR-30 schedule, with the feedback function on the VI+ schedule organized as described in the introduction. The subjects had to depress the lever in both components with a force of $0.343 \mathrm{~N}$ (the same as that in the reports by Reed et al., 2000). The experiment was run for 18 sessions, where each session lasted for $60 \mathrm{~min}$. During the session, the components of the multiple schedule were presented as described above (i.e., six presentations of each component, 5 min per component, 30 -sec intercomponent interval, random order of components across the session). Half of the rats received the VR component on the left lever and the VI+ component on the right lever; the other rats received the reverse organization of components to the two levers.

\section{Results and Discussion}

Figure 2 displays the mean response rates for each three-session block of training for both the VR and the $\mathrm{VI}+$ components of the multiple schedule. An inspection of these data reveals a higher rate of responding on the VR component, relative to the VI+ component. This difference increased across the course of training. These data were analyzed using a two-factor, repeated measures ANOVA, with schedule (VR vs. VI+) and block as factors. A rejection criterion of $p<.05$ was adopted for this and all the subsequent analyses. This analysis revealed statistically significant main effects of schedule $[F(1,7)=54.41]$ and block $[F(5,35)=4.08]$. There was also a significant interaction between schedule and block $[F(5,35)=4.73]$.

The mean reinforcement rates for both components of the multiple schedule were calculated over each threesession block. These rates of reinforcement were always higher in the VR than in the VI+ component. The mean number of reinforcers per minute across all three-session blocks were $\mathrm{VR}=2.4$ and VI+ $=1.0$. These data were analyzed using a two-factor ANOVA (schedule $\times$ block). There were statistically significant main effects of schedule $[F(1,7)=131.79]$ and block $[F(5,35)=4.63]$. There was a significant interaction between schedule and block $[F(5,35)=5.91]$.

The mean reinforced IRTs on each three-session block were calculated. These were lower across all blocks of training for the VR component than for the VI+ component. The mean reinforced IRT for the two components across all three-session blocks were VR $=0.23 \mathrm{sec}$ and $\mathrm{VI}+=2.25 \mathrm{sec}$. These data were analyzed using a two-

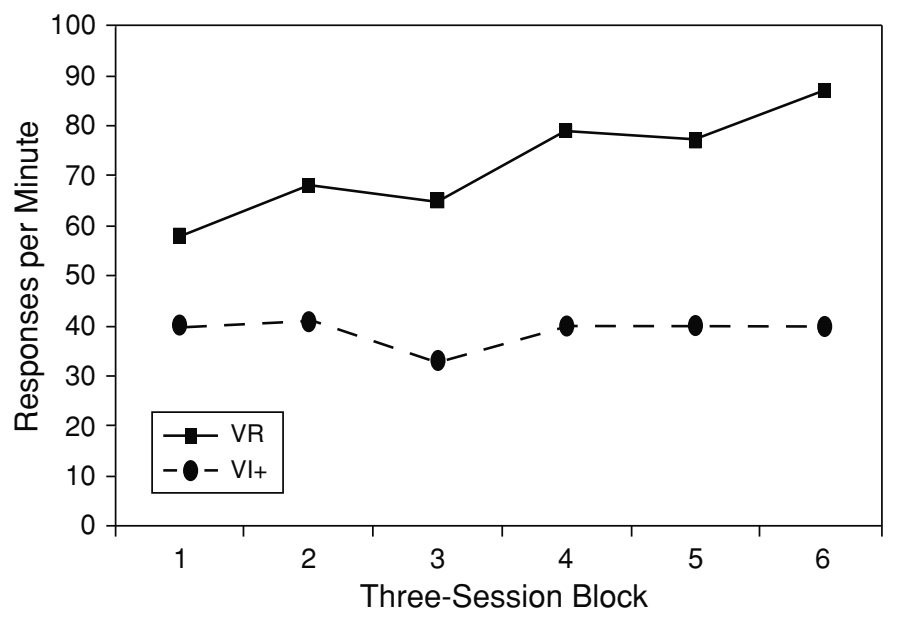

Figure 2. Results from Experiment 1: Response rates during all 6 three-session blocks of the experiment using a multiple VR VI+ schedule. VR, variable ratio 30 ; VI+, variable interval with a feedback function equivalent to that on a VR-30 schedule. 
factor ANOVA (schedule $\times$ block), which revealed only a statistically significant main effect of schedule $[F(1,7)=$ $15.58]$.

These data replicated the findings reported for rats by Reed et al. (2003; Reed et al., 2000) and by Cole (1999); response rates on the VR schedule component were higher than those on the VI+ schedule component. The pattern of data differs from that found for humans, in which the response rates noted on VR and VI+ schedules were similar to one another (McDowell \& Wixted, 1986; Reed, in press). A difference was found also in the present study between the reinforced IRTs for these schedules; reinforced IRTs were shorter on the VR schedules than on the VI + schedules. Although compatible with a molecular analysis, these data do not unambiguously support such a position, since the rates of reinforcement obtained on the two schedules were different. Whatever the explanation for these data, it is clear that the response rate difference between VR and VI+ schedules, previously noted in a between-subjects procedure, can be found in a withinsubjects procedure in rats.

\section{EXPERIMENT 2}

As was noted in the introduction, the discrepancy between the rat and the human data may have resulted from too high a response force requirement being employed with the rat subjects to allow them to maintain responding at a high enough rate to contact the molar aspects of the schedules. Experiment 2 investigated the influence of required response force by replicating Experiment 1 but decreasing the weight of the levers in a low-force condition and increasing the weight of the levers in a high-force condition. If this manipulation affected the rats' sensitivities to the positive feedback function, as was suggested above, the subjects should show similar response rates in the VR and VI+ components with the low response force. In contrast, with high force requirements, response rates should be higher in the VR components than in the VI+ components. Such a finding would serve to highlight a feature of the data so far reported for humans and rat subjects that would explain the discrepancy in the findings (cf. Cole, 1999; McDowell \& Wixted, 1986).

\section{Method}

Subjects and Apparatus. Sixteen male Sprague Dawley rats served in the present experiment. The subjects were 4 months old at the start of training, had a free-feeding body weight range of $330-425 \mathrm{~g}$, and were maintained at $85 \%$ of this weight throughout the experiment. Unfortunately, 1 rat became ill during the course of the study, and the data from this animal were discarded. The subjects had an experimental history similar to that described in Experiment 1 and were maintained as described in Experiment 1. The apparatus was that described in Experiment 1.

Procedure. The subjects were magazine and leverpress trained as described in Experiment 1. They then received 14 sessions of mult VR, VR training, as described in Experiment 1. The subjects were then randomly assigned to two groups (Group Low, $n=8$; Group High, $n=7)$. The pretraining was conducted with a response force of $0.343 \mathrm{~N}$ on both levers for all the animals.

Following pretraining, both groups were exposed to a mult VR-30, $\mathrm{VI}+$ schedule, as described in Experiment 1. The group with a high response force (Group High) had to depress the lever with a force of $0.6 \mathrm{~N}$. Group Low had a required response force of $0.007 \mathrm{~N}$. The experiment lasted for 18 sessions, as described in Experiment 1.

\section{Results and Discussion}

Figure 3 shows the group-mean response rates over each three-session block of training. An inspection of these data reveals that Group Low produced higher response rates than did Group High. Group High had a higher rate of response in their VR component than in their VI+ component. However, there was little difference between the response rates on the VR and the VI+ schedules in Group Low. These data were analyzed using

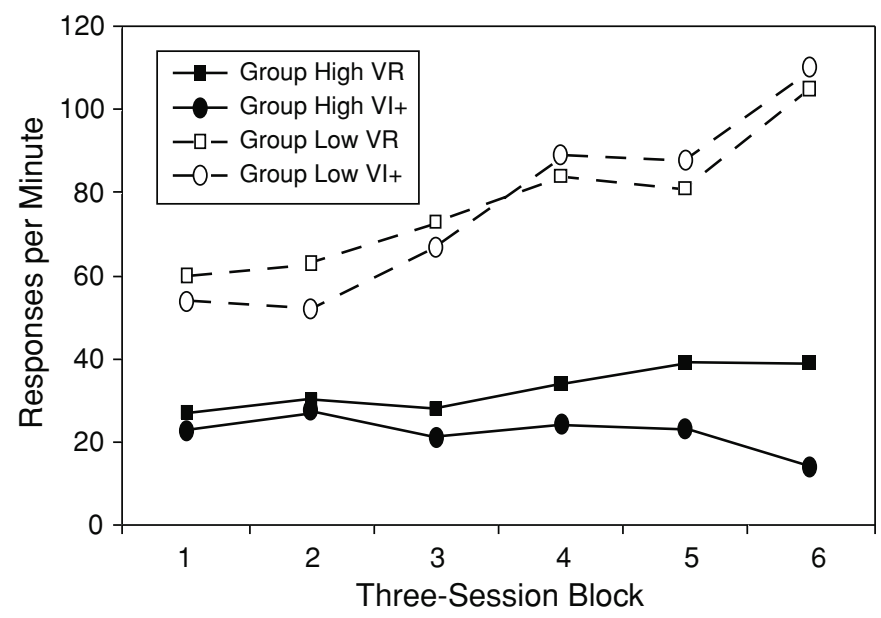

Figure 3. Results from Experiment 2: Response rates for both groups during all 6 three-session blocks of the experiment with a multiple VR VI+ schedule. Group High, response force of $0.6 \mathrm{~N}$; Group Low, response force of $0.07 \mathrm{~N}$. VI+, variable interval with a feedback function equivalent to that on a VR-30 schedule; VR, variable ratio 30 . 
a three-factor, mixed model ANOVA, with force (high vs. low) as a between-subjects factor and schedule (VR vs. $\mathrm{VI}+$ ) and block as within-subjects factors. This analysis revealed statistically significant main effects of force $[F(1,13)=142.39]$ and block $[F(5,65)=33.47]$. There were statistically significant interactions between force and block $[F(5,65)=30.81]$ and between schedule and block $[F(5,65)=6.40]$. No other main effects or interactions were significant. To further analyze the significant interactions, separate two-way ANOVAs were performed on both groups (see Howell, 1997). The two-way ANOVA (schedule $\times$ block) for Group High revealed a statistically significant main effect of schedule $[F(1,6)=24.65]$ and a statistically significant interaction of schedule and block $[F(5,30)=18.71]$. The analysis for Group Low revealed only a statistically significant main effect of block $[F(5,35)=60.38]$.

The group-mean reinforcement rates for both schedule components over each three-session block were calculated. These reinforcement rates were higher for Group Low than for Group High. There was a higher rate of reinforcement for the VR component, relative to the VI+ component, for Group High but no difference between the components in Group Low. The rates of reinforcement (per minute) for the components in each group were the following: Group High, VR $=1.03$ and VI $+=0.51$; Group Low, VR $=$ 1.45 and $\mathrm{VI}+=1.29$. These data were analyzed using a three-factor ANOVA (force $\times$ schedule $\times$ block), which revealed statistically significant main effects of force $[F(1,13)=41.83]$, and schedule $[F(1,13)=6.95]$ and a statistically significant interaction between schedule and block $[F(5,65)=11.45]$. No other main effects or interactions were statistically significant.

The group-mean reinforced IRTs on each three-session block were calculated for each component of the multiple schedule. These data revealed similar short reinforced IRTs for the VR components in both the groups and similar longer reinforced IRTs for the VI+ components for both groups. The mean reinforced IRTs across all three-session blocks were the following: Group High, $\mathrm{VR}=0.34 \mathrm{sec}$ and $\mathrm{VI}+=3.04 \mathrm{sec}$; Group Low, VR $=0.04 \mathrm{sec}$ and VI $+=$ $0.23 \mathrm{sec}$. These data were analyzed using a three-factor ANOVA (force $\times$ schedule $\times$ block), which revealed statistically significant main effects of force $[F(1,13)=20.40]$, schedule $[F(1,13)=21.58]$, and block $[F(5,65)=2.57]$. There were statistically significant interactions between force and schedule $[F(1,13)=15.80]$ and between force and block $[F(5,65)=2.38]$. None of the other interactions proved to be statistically significant.

The results from this experiment demonstrated a clear influence of response force on rates of responding. Response rates were higher for the low-force group than for the high-force group. This is in line with expectation and previous findings on operant conditioning (see Kurtz, 1970; Sumpter et al., 1998). The rates of responding to the VR and VI+ components differed in the two force conditions, as was predicted in the introduction to this study. That is, in the high-force group, where response rates were lower, there was a difference between the response rates to the VR and the VI+ schedules; the VR schedule produced a higher rate of response than did the VI+ component. As with the present Experiment 1, this finding replicated previous work with rat subjects (see Cole, 1999; Reed et al., 2003; Reed et al., 2000). However, in the low-force group, with higher response rates, responding was similar to both the VR and the VI+ components. This finding replicated those previously noted with the generally higher responding human subjects (McDowell \& Wixted, 1986).

The response rate difference in the high-force group was consistent with the differences both in the reinforcement rate (which was higher in the VR component) and with the reinforced IRTs (which were lower in the VR component). However, the lack of difference between the VR and the $\mathrm{VI}+$ response rates in the low-force condition appears inconsistent with either the difference in reinforcement rate or the difference in reinforced IRTs.

\section{EXPERIMENT 3}

The aim of Experiment 3 was to replicate the effect of response force on rats' performance; however, in this experiment, a between-subjects design was employed in order to enhance compatibility with previous rat studies (e.g., Reed et al., 2003; Reed et al., 2000). This was thought to be important, since the response forces used in Experiment 2 had not previously been used with a between-subjects design. It is known that different patterns of results are often found on simple versus complex schedules as a result of simple manipulations (e.g., Reed, 1991).

Four groups of rats were trained in Experiment 3. Two groups were trained on a VR schedule, and two on a VI+ schedule. One VR group and one VI+ group received a high-force requirement, and one group on each schedule received a low force requirement. It was expected, given the results of Experiment 2, that there would be higher rates on the VR schedule than the VI+ schedule for the high-force condition but that the rates would be similar in the low-force condition.

\section{Method}

Subjects and Apparatus. Thirty-two male Sprague Dawley rats served in the present experiment. The subjects were 6 months old at the start of training, had a free-feeding body weight range of $380-470 \mathrm{~g}$, and were maintained at $85 \%$ of this weight throughout the experiment. The subjects had a previous history similar to that described in Experiment 1 and were maintained as described in Experiment 1. The apparatus was that described in Experiment 1, except that only the left lever was inserted into the chamber and no houselights were employed.

Procedure. The subjects received 2 sessions of magazine training on a VT 60 -sec schedule. They then received two 20-min sessions of a CRF schedule. All the subjects were then transferred to a VR schedule for 14 sessions. Each of these sessions lasted for $30 \mathrm{~min}$, and the VR value was gradually increased: Session 1, VR-5; Session 2, VR-10; Sessions 3-5, VR-15; Sessions 6-9, VR-20; Sessions $10-14, \mathrm{VR}-25$. All pretraining was conducted with a response force requirement of $0.343 \mathrm{~N}$. The subjects were then randomly assigned to four equally sized groups.

Two groups were exposed to a VR-30 schedule; one of these groups had a high response force requirement (Group VR-High), and the other had a low response force requirement (Group VR- 
Low). The other two groups of rats were exposed to a VI+ schedule, so that the feedback function relating the rate of response to the rate of reinforcement was the same as that on a VR-30 schedule, as described in the introduction. One of these groups had a high response force requirement (Group VI+-High), and the other had a low response force requirement (Group VI+-Low). The groups with a high response force had to depress a lever with the force of $0.6 \mathrm{~N}$, in comparison with a required force of $0.007 \mathrm{~N}$ for the groups with a low response force.

The experiment was run for 18 sessions, where each session lasted for $30 \mathrm{~min}$.

\section{Results and Discussion}

Figure 4 shows the group-mean response rates over each three-session block of training. Group VR-High had a higher rate of responding than did Group VI+-High. However, there was less of a difference between the response rates of Group VR-Low and Group VI+-Low. These data were analyzed using a three-factor, mixed model ANOVA, with schedule (VR vs. VI+) and force (high vs. low) as between-subjects factors and block as a within-subjects factor. This analysis revealed a statistically significant main effect of block $[F(5,135)=6.74]$. There was also a significant interaction between schedule and block $[F(5,135)=4.61]$. No other main effects or interactions were significant.

To further analyze the interaction and what was apparent from an inspection of Figure 4, separate two-way ANOVAs (schedule $\times$ block) were performed on each of the force conditions. This analysis for the high-force groups revealed statistically significant main effects of schedule $[F(1,14)=7.67]$ and block $[F(5,70)=2.56]$ and a significant schedule $\times$ block interaction $[F(5,70)=$
2.52]. The analysis for the low-force group revealed only a statistically significant effect of block $[F(5,65)=6.61]$.

The group-mean reinforcement rates over each threesession block were calculated. These were higher for the VR groups than for the VI+ groups. The mean number of reinforcers per minute across all 6 three-session blocks were the following: Group VR-High = 1.8; Group VR-Low = 2.6; Group VI+-High $=0.7$; Group VI+-Low $=1.1$. These data were analyzed using a three-factor ANOVA (schedule $\times$ force $\times$ block). There was a statistically significant main effect of schedule $[F(1,27)=12.58]$. No other main effects or interactions were statistically significant.

The group-mean reinforced IRTs on each three-session block were calculated for each group. These data showed similar short reinforced IRTs for both of the groups responding on the VR schedules and similar longer reinforced IRTs for both of the groups on the VI+ schedules. The mean reinforced IRTs across all 6 three-session blocks for the groups were the following: Group VR-High = $0.6 \mathrm{sec}$; Group VR-Low $=0.4 \mathrm{sec}$; Group VI+-High $=$ $5.1 \mathrm{sec}$; Group VI+-Low $=3.1 \mathrm{sec}$. These data were analyzed using a three-factor ANOVA (schedule $\times$ force $X$ block), which revealed only a statistically significant main effect of schedule $[F(1,27)=20.60]$. No other main effects or interactions were statistically significant.

The results from this experiment demonstrated that the high-force groups responded more quickly on the VR schedule than on the VI+ schedule. This pattern of data was similar to that found in the present Experiments 1 and 2 with a high response force requirement, in which, however, a within-subjects design was used. These patterns are similar also to those reported by Cole (1999) and by

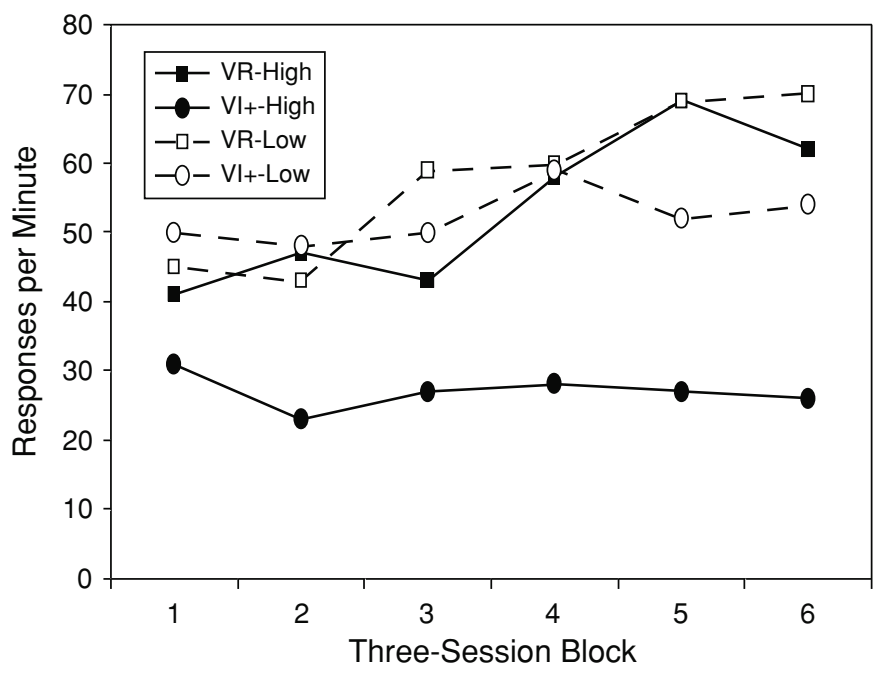

Figure 4. Results from Experiment 3: Response rates for all groups during all 6 three-session blocks of the experiment. VI+-High, variable interval with an equivalent feedback function equivalent to that on a VR-30 schedule and a response force of $0.6 \mathrm{~N}$; VI+-Low, variable interval with a feedback function equivalent to that on a VR-30 schedule and a response force of $0.07 \mathrm{~N}$; VR-High, variable ratio 30 with a response force of $0.6 \mathrm{~N}$; VR-Low, variable ratio 30 with a response force of $0.07 \mathrm{~N}$. 
Reed et al. (2003; Reed et al., 2000), using rat subjects. In contrast, in the low response force requirement conditions, rates of response were similar in the two schedule conditions. This pattern of results replicates those seen in the present Experiment 2 with low-force requirements with a within-subjects design and those noted in human subjects (McDowell \& Wixted, 1986; Reed, in press). As with the present Experiment 2, the response rate difference between the VR and the VI+ schedules in the highforce conditions is compatible with either a reinforcement rate or a reinforced IRT account. However, the lack of difference in response rate between these two schedules noted in the low-force conditions is at odds with the difference in reinforcement rate and reinforced IRTs across the schedules. Whatever the explanation for this finding, it appears to corroborate what was noted in the present Experiment 2.

\section{GENERAL DISCUSSION}

In this series of experiments, VI+ and VR schedules were employed, and the response rates, reinforcement rates, and reinforced IRTs on the two schedules under both high and low response force requirement conditions were compared. This manipulation was conducted in order to resolve the discrepancy between the results noted in previous studies employing VR and VI+ schedules. McDowell and Wixted (1986; see also Reed, 1999, 2001, in press) found no difference between VR and VI+ schedules with humans, whereas Cole (1999) and Reed et al. (2003; Reed et al., 2000) found higher VR than VI+ response rates with rat subjects. It was suggested that if the force requirement in the studies in which rat subjects were used had been made less stringent, rates of responding might have been able to be maintained long enough to contact the molar aspects of the contingency; thus, the VR versus VI+ response rate difference should be abolished with low-force requirements.

In Experiment 1, a novel within-subjects procedure with rats was used, and the VR versus VI+ response rate difference previously noted using between-subjects designs was replicated. This result was entirely consistent with Cole's (1999) and Reed et al.'s (2003; Reed et al., 2000) findings. A similar higher VR than VI+ rate was noted with a between-subjects design in Experiment 3, using a high response force requirement. In contrast, in Experiment 2 (within subjects) and Experiment 3 (between subjects), a much reduced response force requirement was associated with only a small VR versus VI+ schedule response rate difference. This similar response rate on the two schedules was noted despite different rates of reinforcement and reinforced IRTs. The latter result is consistent with the findings reported for human subjects by McDowell and Wixted (1986).

That the response force manipulation was successful can be seen in a direct comparison between the high- and low-force conditions in Experiments 2 and 3. However, it can also be seen in a cross-experimental comparison between the two within-subjects procedures (comparison with Experiment 3 is made difficult, due to the use of a between-subjects procedure and the use of 30-min, rather than 60-min, sessions in the latter experiment). Terminal rates of response per minute on the VR schedule at the different response force conditions were the following: VR schedule, $0.07 \mathrm{~N}=110,0.343 \mathrm{~N}=86,0.6 \mathrm{~N}=38$; VI+ schedule, $0.07 \mathrm{~N}=105,0.343 \mathrm{~N}=40,0.6 \mathrm{~N}=$ 13. This finding replicates previous demonstrations of response force requirements on instrumental responding (see Kurtz, 1970; Sumpter et al., 1998). The ratio of VR to VI+ rates of responding can also be seen to increase as a result of increasing response force: $0.07 \mathrm{~N}=$ 1.0 (Experiment 2) and 1.3 (Experiment 3); $0.343 \mathrm{~N}=$ 2.1 (Experiment 1); $0.6 \mathrm{~N}=2.9$ (Experiment 2) and 2.4 (Experiment 3). This analysis shows what is apparent in the present experiments, that under low response force conditions, rats come to respond similarly on VR and VI+ schedules, in a way that they do not under high response force requirement conditions.

Thus, it is clear that the present experiments demonstrated that the pattern of data reported by McDowell and Wixted (1986; see also Reed, in press) could be replicated with rats under low response force conditions. However, with higher response force conditions, the VR versus VI+ response rate differences noted previously with rats were replicated (see Cole, 1999).

The issue remains as to why manipulation of response force produces these different results. One possibility concerns the ability of the contingency to sustain high rates for the particular species exposed to that contingency. In the studies with human subjects (i.e., McDowell \& Wixted, 1986; Reed, in press; see also Reed, 1999, 2003), the rate of responding has been considerably higher than that noted in nonhuman subjects (i.e., Cole, 1999; Reed et al., 2003; Reed et al., 2000). It may be that sensitivity to the VI+ schedule emerges only when it is possible to maintain a high rate of responding across the session. Lowering the response force requirement may allow the rat subjects to achieve this high rate of responding. Certainly, the VR versus VI+ rate difference does appear to emerge with human subjects when the response force is increased (see Reed, in press, Experiment 1).

There are a number of reasons why response rate may matter in the generation of these results. It may be that higher response rates allow the differential reinforcement of short IRTs. In turn, this would generate high overall rates of responding. There is no doubt that selective reinforcement of IRTs does alter performance on VI+ schedules (see Reed et al., 2003; Reed et al., 2000). However, the present results suggest that under the low force requirement conditions, equal rates of response on the VR and $\mathrm{VI}+$ schedules were generated in the absence of similar reinforced IRTs. In fact, the IRTs on the VI+ schedule were longer than those on the VR schedule (see also McDowell \& Wixted, 1986). Of course, it may be that it is not the single IRT emitted just prior to reinforcement that is critical but, rather, the local rate of responding just prior to reinforcement. The latter rate might have been more similar in the current VR and VI+ schedules than the terminal 
reinforced IRT (but unfortunately, this local rate was not measured in the present series of experiments). There is evidence that manipulation of local rates is important in generating response rate effects (see Reed, 1989, 2003; Wearden \& Clark, 1988). However, in the absence of further data, this account is speculative.

The suggestion by Cole (1999; see also Reed et al., 2000) that the functioning of the VI+ schedule makes any pause in responding potentially catastrophic for local reinforcement rates warrants consideration in this context. A reduction in the rate of response on the VI+ schedule will generate an increased interreinforcement interval, which, if it becomes too long, will lead to extinction. If rates are not maintained across the schedule, this could dramatically reduce the response rates seen on the VI+ schedules. Such a catastrophic drop in responding was seen in some rats studied by Reed et al. (2003) when they were transferred to a VI+ schedule from a VR schedule. The use of low force requirements may enable rat subjects to continue to emit high rate across the session and not be subject to these local extinction effects, although it should be noted that rates on VR and VI+ schedules continued to differ when Cole attempted to utilize a schedule manipulation that overcame this local extinction effect. However, there are few data by which to see whether this manipulation actually worked.

Finally, it is possible that the high rates of responding allowed true sensitivity to the molar characteristics of the $\mathrm{VI}+$ schedule. With high rates, it is possible to allow differential conditioning of the rates, in a way that low rates would not allow this reinforcement. A greater range of response rates would allow a greater sampling of the correlation between response and reinforcement rates, and this may facilitate the acquisition of control over responding by the molar features of the environment. The low response force requirement would allow such high rates.

The reason why human subjects display and maintain higher response rates than do rat subjects with such apparent ease is unclear. It could be that human subjects form verbal rules for themselves that allow this to occur (see Lowe, 1979). Human subjects could be capable of faster responding than rats, and pigeons' keypecking may provide data comparable to those for humans, since this response can be emitted at high rates. Alternatively, it could be that there is less scope for competing behavior in the human experimental situation, in comparison with the nonhuman situation.
Whatever the eventual explanation of the issues above, the present data suggest that it is a procedural, rather than a species, difference that produces the differing results with respect to VR and VI+ schedules, and they also constitute the first data suggesting rat sensitivity to VI+ feedback functions.

\section{REFERENCES}

Cole, M. R. (1999). Molar and molecular control in variable-interval and variable-ratio schedules. Journal of the Experimental Analysis of Behavior, 71, 319-328.

Howell, D. C. (1997). Statistical methods for psychology. New York: Duxbury.

Kurtz, K. H. (1970). Food deprivation and effort expended for food. Learning \& Motivation, 1, 281-296.

Lowe, C. F. (1979). Determinants of human operant behavior. In M. D. Zeiler \& P. Harzem (Eds.), Advances in analysis of behavior: Vol. 1. Reinforcement and the organisation of behavior (pp. 159-192). Chichester, U.K.: Wiley.

McDowell, J. J., \& WiXted, J. T. (1986). Variable-ratio schedules as variable-interval schedules with linear feedback loops. Journal of the Experimental Analysis of Behavior, 46, 315-329.

REED, P. (1989). The influence of interresponse time reinforcement on the signalled-reward effect. Journal of Experimental Psychology: Animal Behavior Processes, 15, 224-231.

ReED, P. (1991). Multiple determinants of the effects of reinforcement magnitude on free-operant response rates. Journal of the Experimental Analysis of Behavior, 55, 109-123.

REED, P. (1999). Effect of perceived cost on judgements regarding the efficacy of investment. Journal of Economic Psychology, 20, 657-676.

REED, P. (2003). The effect of signaled reinforcement on rats' fixedinterval responding. Journal of the Experimental Analysis of Behavior, 79, 367-382.

REED, P. (in press). Human sensitivity to reinforcement feedback functions. Psychonomic Bulletin \& Review.

Reed, P., Hildebrandt, T., DeJongh, J., \& Soh, M. (2003). Rats' performance on variable-interval schedules with a linear feedback loop between response rate and reinforcement rate. Journal of the Experimental Analysis of Behavior, 79, 157-173.

Reed, P., Soh, M., Hildebrandt, T., DeJongh, J., \& Shek, W. Y. (2000). The influence of feedback on molar and molecular contingencies: The variable-interval-plus-linear feedback-loop schedule. Journal of the Experimental Analysis of Behavior, 82, 246-255.

Sumpter, C. E., Temple, W., \& Foster, T. M. (1998). Response form, force, and number: Effects on concurrent-schedule performance. Journal of the Experimental Analysis of Behavior, 70, 45-68.

WEARDEN, J. H., \& CLARK, R. B. (1988). Interresponse-time reinforcement and behavior under aperiodic reinforcement schedules: A case study using computer modeling. Journal of Experimental Psychology: Animal Behavior Processes, 14, 200-211.

(Manuscript received August 4, 2004; revision accepted for publication May 11, 2006.) 